\title{
The status of Chinese handwriting identification and the improvement of methodologies
}

\author{
Guanqing Wang* \\ Criminal Justice College of East-China University of Political Sciences and law, 555 Longyuan Rd, Shanghai China
}

\begin{abstract}
The testimony of handwriting identification is more uncertain than other kinds of physical evidence identification. If the expert did not employ the principles and methodologies based on neurophysiology, the examination would not be sufficient, and the testimony might be suspect. This research describes how neurophysiology influences the feature of handwriting and the methodology of identification: most handwriting characteristics have high-level individualization determined by the nervous system. The methods of defining the characteristics of handwriting were explored and it was found that writing motion rules and writing norms are effective measures to compare and match writing characteristics. Additionally, the number of characteristics and their degree of individualization are the bases to assess the handwriting characteristics system, quantitatively and qualitatively. Practical cases have been presented to support the theories proposed.
\end{abstract}

\section{Introduction}

Experts generally regard that writing is brain-based, by the phenomenon that once a person owns the writing skill, whether he/ she writes with the dominant hand, weaker hand, mouth, or foot, most of the characteristics are the same [1]. One's writing process involves cognition, kinesthetic, and manual skills [2]. Most experts also agree that uniqueness and natural variation are two crucial fundamental principles of handwriting identification [3]. That any person who observed another person's writing was permitted to testify respecting the authenticity of a writing, despite the inherent weaknesses in this evidence [4]. These theories are currently the basic recognition of handwriting identification.

\section{Methods \\ It is generally regarded that identification was based on dynamic features associated with gestures and movements of writers [5]. Considering the variability of handwriting, understanding how people write is key in the development of handwriting examination systems. Although numerous models have been proposed to study and analyze handwriting [6], there is a consensuses that handwriting analysis starts with objective factors such as speed, size, shape, slant, and symbolic feature, and the combination of analysis of these elements produces a full personality profile $[7,8]$. With the development of computer technology, some new methods for digitized analysis had been proposed. However, there was no report that these methods were generally accepted. Manual analysis is still the main method of handwriting identification.}

In practice, researchers had conducted a study to test the proposition that professional document examiners possess writeridentification skills absent in the general population. As shown by an experiment: non-professionals mismatched $38.3 \%$ documents created by different writers, while the professionals' mismatching rate is $6.5 \%$ [9]. Another similar research found that Type II error was made by forensic document examiners at the rate of $7.05 \%$ of the cases, and laypersons $26.1 \%$ [10]. The methods used by non-professionals were generally to estimate grossly, and the professionals' method was not essential exclusiveness, compared to laypersons.

\section{The status quo analysis of handwriting identification}

\section{The rate of false identification is still high}

In practice, some methods were developed for forging and disguising handwriting. But generally, each expert's methods are different, more or less. Experts sometimes could not recognize forged or disguised handwriting when it looked normal and natural, for the expert doesn't understand the rules of writing motions and their neurophysiological features. Handwriting characteristics are determined by the physiology system, so authors have quite limited ability to manipulate the characteristics. The activity of forging or disguising handwriting is controlled psychologically and only a small portion of characteristics in the system can be altered. The degree of forging or disguising is proportional to the author's writing skill level. For one's normal writing practice, the writing ability is proportional to the degree of writing complexity and difficulty. The degree of writing fluency is formed by both this ability and the practice of writing. Figuring out the author's original characteristics and their individualization is based on the writing ability defined by fluent writing motions.

Experts should continue focusing on faked documents and keep up to date on the developing tendency in judicature to be familiar with perjurers' behaviors. Experts should also realize that perjurers research their identification methods and capability. In China, perjury in civil litigation will not generally be deemed a crime, so some perjurers were

${ }^{\star}$ Correspondence to: Guanqing Wang, Criminal Justice College of East-China University of Political sciences and law, 555 Longyuan Rd, Shanghai China, Tel: 014756558806; E-mail: wangguanqing100@gmail.com

Keywords: handwriting characteristics, physiological habits, methods of identification

Received: May 06, 2019; Accepted: May 15, 2019; Published: May 18, 2019 
businessmen who had undergone litigation more than once. Knowing that experts could not identity some forged documents will encourage them to commit perjury in the subsequent lawsuit by forging or disguising handwriting.

The following are two types of disguising signature in documents:

Cases of guaranteed contract: The perjurer's company is starved for cash and wants to get a loan from a financial institution. The owners of the company would be required to guarantee the debt with personal property. He/she doesn't want to be bankrupted later if their company is unable to pay off the loan. Before signing the guarantee contract, he/she might have designed the signature and practiced until it looked natural. And they obviously can't change their signature, for the representative may be familiar with their signature style and will deny the loan for the disparity. Due to the same signature style and the different parts with natural figure, many experts considered it as a mimic signature and drew a false conclusion.

Cases of labor contract: The labor contract law of the People's Republic of China (2013 edition) prescribes that if a company doesn't sign a labor contract, it would pay double salary to the employee as penalty. Since this law was issued, there have been some workers who coveted the double salary. When they signed the contract, they used a kind of signature model which they had never signed on other documents. After they had worked for several months, they sued the company for compensation by claiming that there was no labor contract. The court commissioned the signature identification to experts but sometimes the expert could not discover the truth.

\section{False experts' opinion can be admitted}

That false experts' opinions are admitted might involve two kinds of situations: disclosure, and selection among conflicting opinions. The first situation occurs when the expert gives a false conclusion while one or both parties of litigants do not actually know the truth. So, the opinion of the expert is admitted in good faith. The second situation occurs when a court invites the expert to identify after the first commission, and the expert's opinion is contrary to the prior identification, and the court selects a wrong opinion for consideration. These two kinds of situations will result in the truth not being discovered, for there are no standards for admitting expert's testimony in courts in China, and cross-examination is not valuable enough either. Whether to admit an expert's testimony is mainly decided by the court.

In 1993, with Daubert v. Merrell Dow Pharmaceuticals, Inc. [11], the U.S. Supreme Court defined a new era in determining the admissibility of expert testimony. In this case, the Court interpreted Rule 702 of the Federal Rules of Evidence that expert testimony is admissible only if it is both relevant and reliable. Federal Rule 702 regulates:

If scientific, technical, or other specialized knowledge will assist the trier of fact to understand the evidence or to determine a fact in issue, a witness qualified as an expert by knowledge, skill, experience, training, or education, may testify thereto in the form of an opinion or otherwise [12].

But in China, the court will not require the expert to offer information regarding their education, skill, experience or training. Quite a number of experts did not receive formal education or adequate training, and litigants cannot get the case information of the expert's prior testimony for challenging the expert's skill and capacity.

\section{The neurophysiological base of handwriting identification}

Many experts viewed the handwriting as images while they were examining. These images may have been the shape of the signature, the style of one character, or the combination of a few letters or parts of a character, so there was no standard and there were no fixed units used for matching the characteristics. Handwriting identification should be to compare the physiological habits system between the questioned document and handwriting sample documents, not to compare the handwriting styles as images. During the examination, this habits system is expressed by the total characteristics in handwriting; however, there were still many examiners who only analyze part or a few characteristics instead of the whole characteristics system, depending on what they found and would weigh. The reason to identify the physiological habits system rather than a similar part, a few habits, or the writing figure is because one will always have some common habits with others, and the same person's handwriting could vary with deliberate writing behavior or for different environments. So, if there are not enough habits to draw a conclusion, the expert's opinion will not be reliable $[13,14]$. However, many experts stated in their reports that they used the identification of habit rather than the physiological habits system.

\section{Handwriting reflects a neurophysiological habits system}

Handwriting is a physiological phenomenon which attributes to the sphere of cognitive science.

Cognitive science is the interdisciplinary study of mind and intelligence, embracing philosophy, psychology, artificial intelligence, neuroscience, linguistics, and anthropology. Its intellectual origins are in the mid-1950s when researchers in several fields began to develop theories of mind based on complex representations and computational procedures [15].

The physiological system that controls personal writing motions will not have enough stability, and the intentional actions will increase the variety of writing. Researchers stated that handwriting is the final response to a complex cognitive and neuromuscular process which is the result of the learning process [16]. The neural system consists of: (1) a set of basic motor engrams for the most common words and groups of letters; (2) a set of rules for combining basic motor engrams into larger units [17]. An experimental research described that the standardized handwriting for providing objective measures to distinguish Parkinson patients from healthy control participants [18]. A study discovered "large, distributed neural populations in human cortex (M1) and anterior intraparietal sulcus (aIPS) encode complex handwriting movements regardless of their particular dynamics and kinematics, in a scale-invariant manner." [19]. An exploratory analysis showed clusters in the left ventral premotor cortex and inferior and superior parietal cortices were only significantly active for right-handwriting while comparing the writing and other activities of the right hand, left hand and right foot [20]. The motor equivalence theory referred to a writing motor program, which stores the writing motion's characteristics in a higher order area rather than the primary motor cortex and can be accessible by all effectors, such as hand, foot, or mouth. From the above researches, it can be deduced that handwriting consists of many motor memory units that form various characters. The same writing habit is expressed for the same writing motion even for handwriting in different languages' handwriting. And this also explains why a person's handwriting in different languages can be compared to identify whether it comes from the same writing habits system (Figure 1).

The object of handwriting identification is the synthetic reflection of one's physiological writing habits system, not the handwriting per se. So, to examination professionals, handwriting appears to be a reflection of the system [21]. Saunders and Buscaglia said that experts 


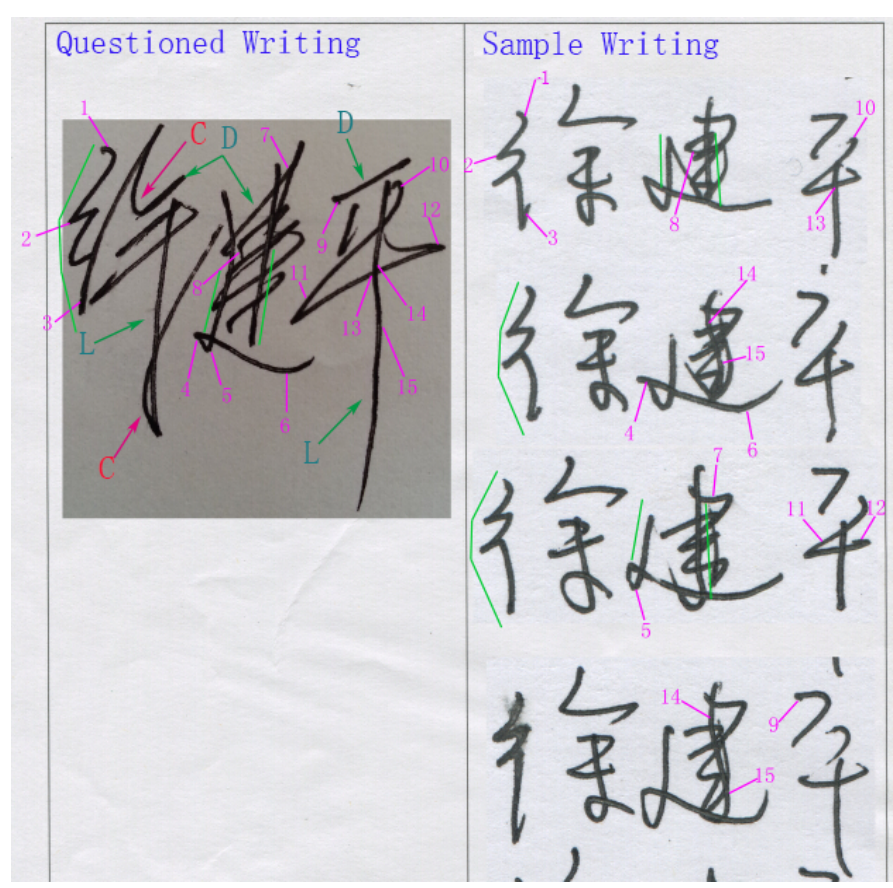

Figure 1. Left signature was from a debt guarantee document, the right signatures were acquired in court for the purpose of comparison. All the marked characteristics are for testing [23]

did regard the proposition - that the writing profiles were unique as a key premise underlying forensic handwriting comparisons. On the other hand, the empirical study cannot validate this proposition due to the impossibility of observing sample documents written by every individual [22]. This explains the reason why experts make mistakes while examining handwriting. Even if they know this conception, they are not really testing the habits system. They can only regard the handwriting as the images. However, when one or a few handwriting characteristics had been changed due to reasons such as disease or disguise writing, the picture of the writing will be changed greatly. The expert would be uncertain whether the handwriting came from another author. That is why some experienced experts want to have more samples, for they hope to find out whether there is a sample that possesses more similar writing figures to the handwriting from the questioned document.

As shown in figure 1, the signature in the questioned document was changed by the curve method (labeled "C"), the length of strokes (labeled "L") and all the crossed writing direction (labeled "D"). All these changes were easily formed intentionally. However, most writing motion characteristics have been retained (as shown in the matched characteristics labeled " $1-15$ "), for it is difficult for the author to control all his/her writing habits. So, the signatures, both the questioned document and the samples, come from the same author, even though the figures of the signatures appear to be entirely different. From this case it can be noted that the figure of handwriting was changed greatly, and it was not appropriate for experts to treat the writing as in the picture while identifying the writing.

The writing habits system is a neurophysiological phenomenon, consisting of many habits that exist in the neural system. Writing motions are very complicated, sometimes subtle and various, which determine the complexity of the writing dynamic stereotype, and every person has a different writing habits system. The writing habits system has a high degree of individualization for the combination of the habits' individualization in author's physiological system [13]. The individualization value is the rate of a habit among people. The number of habits and their individualization make handwriting identification possible, for there are quite a lot of writing motion habits, and many of them have a high-level degree of individualization. The writing habits system is the objective of identifying handwriting, and it is also the basis for analyzing forged and disguised handwriting.

\section{The features of handwriting characteristics and habits system}

Through a statistical analysis of 500 writing samples with a total of approximately 180,000 Chinese characters, general writing rules were found in the sampling. More than 200 characteristics have been defined, and it was found that their individualization is determined by the neural system or the writing convenience. Most characteristics are correlated with writing norms. When a norm is hard to conform, the writing form that can obey it will have a higher degree of individualization. That is, the degree of individualization is positively related to compliance with writing norms. When a norm is easy to conform, the writing style's individualization is negatively related to the compliance level. Sometimes the individualization of a characteristic is influenced by the convenience in writing. People tend to adopt easier styles to write for convenience. The easier a writing mode is to implement, the less possible to individualize it, and vice versa. Generally, most characteristics have a high degree of individualization and are not heavily correlated to the convenience of writing. So, it can be speculated that individualization is decided by the features of neurophysiology. However, measurements have not been discovered to quantify the specific causality between characteristics individualization and the mechanisms of neurophysiology [13]. But this doesn't hamper these rules used in instructing professionals to analyze the individual level of characteristics and the individualization of the physiological habits system. These rules were implemented in practice and proved feasible. And during the application, it was found that the forged writing style actually was the writer's own habits which are used in other characters [24]. This is illustrated by the case of the “郑宾” signature in figures 6 and 7. The theories of habits system can explain that the author cannot write the characteristics outside his/her habits system with a normal writing style, since a person can only write within their inherent habits.

\section{How should experts treat the handwriting while conducting identification?}

The expert should remember: each writing expresses an author's habits system, wholly or partly depending on the quantity of the writing. Part of the habits system can also be drawn from the conclusion of uniqueness, for handwriting characteristics by motion units can be the abundant in a few characters, and it will be qualified to analyze the feature of neurophysiological habits system. However, there are still some experts who think handwriting is a psychological phenomenon reflecting the psychological characteristics [25]. Actually, one can only control a limited number of characteristics, as showed in the above cases.

\section{Methodologies of improving handwriting identification}

\section{The qualities requirement of the experts}

Knowledge base. An expert should have the knowledge of physiology so that he/she can understand the features of writing motions and pay attention to the individualization of the physiological habits system rather than the figure of writing. One's knowledge will determine his 
direction of work. A few hours of physiology training is not helpful to guide the expert to analyzing handwriting physiologically. Possessing integrated knowledge in physiology or medical science will greatly advance one's analytical skills. An expert should also accumulate and update their social knowledge, including criminal behavior that relates to forged documents, for new policies or economic development will incur the era hallmark of fraud and special methods of evidence forgery. The expert will also be more alert to forged documents, and that will benefit the expert's practice.

Research skills. An expert who is distinguished in practice, usually has conducted a lot of researches in handwriting identification. In spite of the physiological base of handwriting, there is not much experimental research about neurophysiological writing motion and characteristics' rules, as it is quite difficult to design research methods for this complex writing phenomenon. However, if an expert wants to excel in this field, studying handwriting physiologically will help him/ her to understand the features of handwriting, whether the research is formal or informal. Physiologically recognizing the characteristics during analyzing writing habits is essential.

Practice skill. An entrant should practice the analysis for many cases until mastering the skills of defining characteristics, assessing the value of each characteristic, and the uniqueness of the entire physiological habits system. His/her attention must be focused on the specific characteristics rather than on the figure of the writing. He/ she should know the quality and quantity of the characteristics which came into view, not over value one or few writing models, and not let these models influence his/her conclusion. Generally, the overall minor motions' characteristics, such as the pressure of the tool or the cross position between two strokes are key for identification because they are the stable expression of the physiological habits system, and hard to be controlled by the author (see the case of “多晓艳” in figure 8 and 9).

\section{Methodologies of selecting sample handwriting}

In practice, it is regarded that comparable handwriting both from the questioned documents and sample documents should be of the same language, and generally experts require the handwriting must be the same script so that it will allow for comparing the characteristics. It is generally regarded while acquiring the sample must consider the preexistent, preconceived, free written documents before the litigation: "If the writing in litigation is a cursive handwriting, the comparison model must be written in cursive handwriting. If the writing in litigation is executed with capital letters, the comparison model must be made with capital letters [26]." Undoubtedly it is easy to compare the characteristics among the same characters. However, we usually do not have a chance to achieve this ideal, for expert cannot force the litigants to offer natural documents or to write according to our requirement. the court may lose the opportunity of finding the truth because of "questioned handwriting lacking identification quality". This generally happens if the expert over valued the consistency of handwriting between the questioned and sample documents. During the prior research of statistics handwriting characteristics, writing motion units which were defined by decomposing the writing style of the characters was used to determine the characteristics or habits [27]. During this research process, it was discovered that if the experts treat handwriting physiologically according to the unit of writing motion, the difference of the language character in handwriting will not be a problem. On one hand, when a person uses different characters, the writing motions will not be changed, since the same author operates with his own stable writing style. On the other hand, if characteristics are considered by the units of writing motion, different characters will have many of the same writing norms. These allow for comparing characteristics among different characters. That experts require the consistency of characters is because they don't understand what to compare, they still treat handwriting to be images. The following case proves it is possible to compare characteristics from different characters (Figures 2-5) [28].

In the above pictures, comparing the characteristics is to match the writing motions, not to match the figures of the characters. Different characters can possess the same structure, the same letter or stroke, and the same writing motions of strokes, etc. So, it is feasible to compare the cursive handwriting with typing writing or writing in English with writing in Chinese. In this situation, it requires more effort and work for the expert. The characteristic unit is the single writing motion style, such as the beginning, the ending and the intermediate movement form of a stroke - they are three basic motion units while comparing a single stroke. The unit should be the smallest single motion. If an expert regards the characters or letters as the units, he/she is not comparing the characteristics but comparing the shape or figures of the characters or letters. And he/she will reduce the number of characteristics greatly. The shape of a character or a letter is only one characteristic, and it can easily be changed on purpose by changing one writing style among the character. Remember that the comparison unit is the writing motion and is very important, This, can help experts come to the objective comparison: defining numerous characteristics and analyzing the quality and quantity of characteristics. Analysis based on the units of characters or letters is actually covering fewer characteristics, and it is

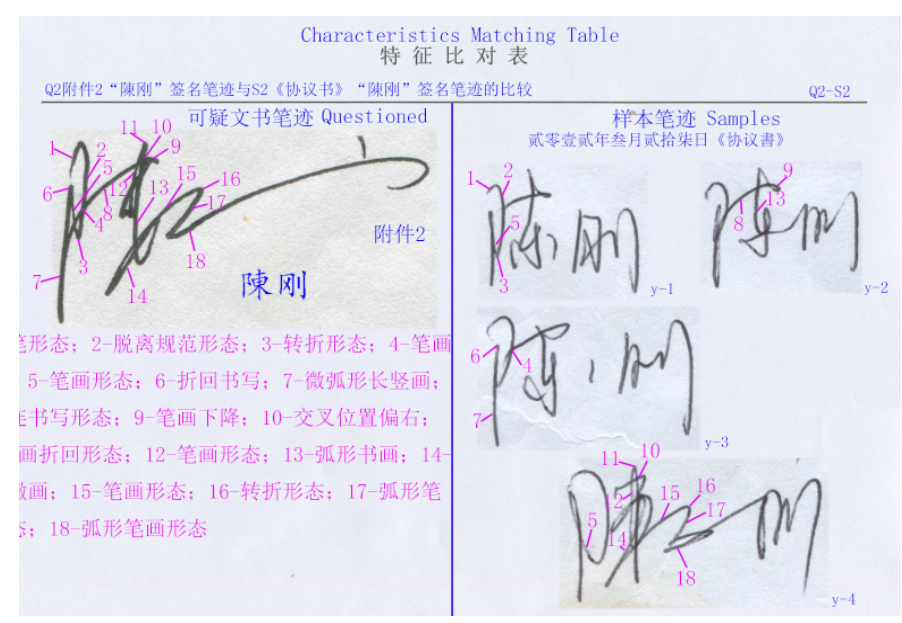

Figure 2. The questioned signature is of different chirography from the sample signatures, but they still have the same structures and same writing motions

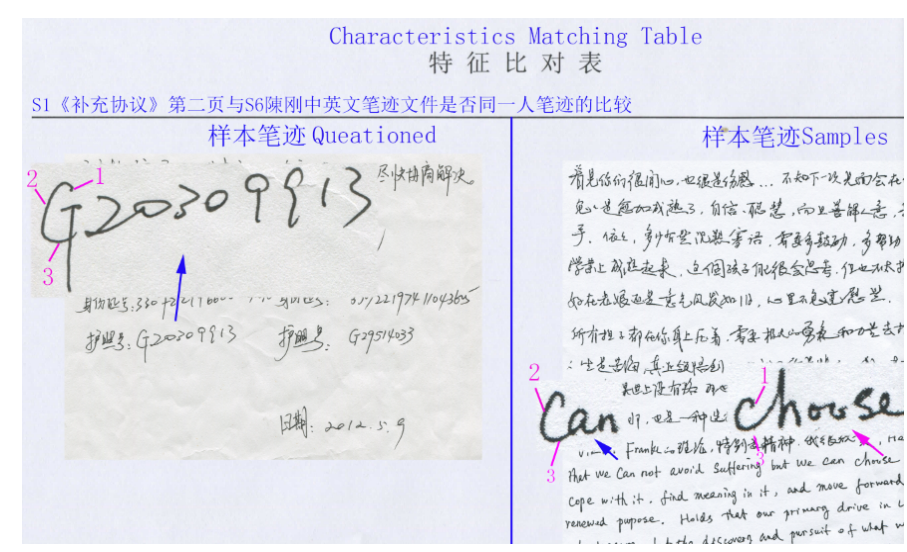

Figure 3. Different letters have the same writing motions 


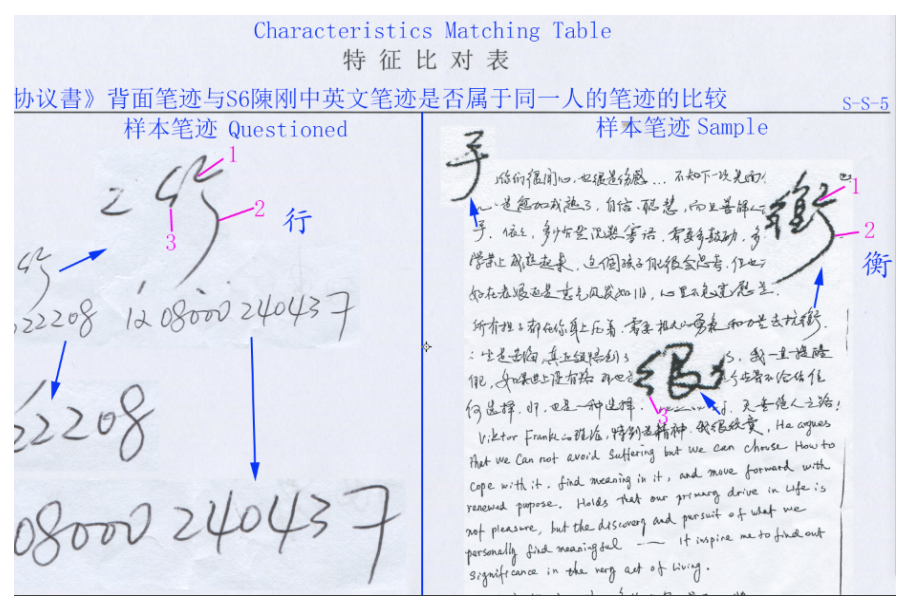

Figure 4. Same strokes expressed same characteristics

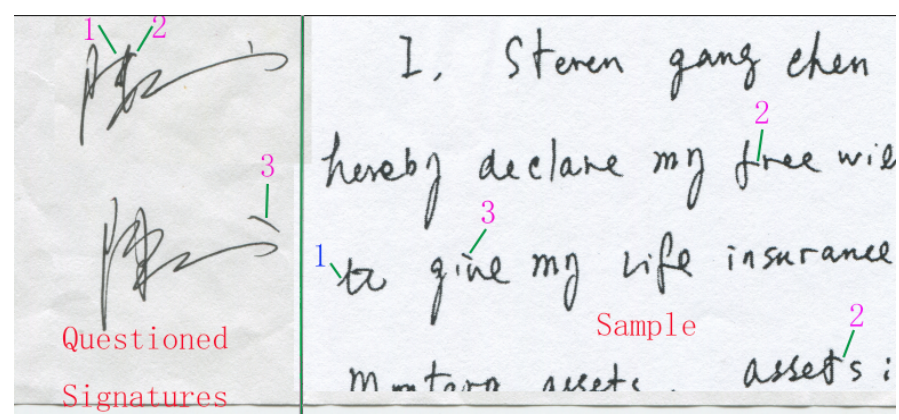

Figure 5. Same writing motions

hard to figure out the quality and quantity of all characteristics. For example, the shape of a letter is unlimited, for each person may write a different shape. It is impossible to demonstrate the individualization of habits system and reach a credible conclusion.

\section{Analysis methods of handwriting motions}

The base of identification: Physiological writing system has its own rules: the same habit will appear in the same structure of the characters or letters [14]. Experts should know this and use it to analyze the characteristics. There are three steps, as follows:

Firstly, by observing the writing of the questioned document and the sample documents to figure out the statuses of their respective habits systems.

Secondly, by the known characteristics to deduce that the same characteristics will necessarily appear when there is more handwriting which possesses the same writing motions, and the entire habits system will be expressed if there is enough handwriting.

Finally, for rare characteristics the expert can define whether they belong to the habits system. If the perjurer wants to let his signature to look natural and "tell" the expert it is actually unnatural by changing some characteristics. His/her writing will be fluent and the changed writing styles usually belong to his/her original habits, which are not only expressed in the signature. This can be seen in the following case (Figures 6 and 7) [29]:

In figure 6, comparing the questioned writing with the sample writing, most minor characteristics are matched. Even though the sample writing was offered during the litigation, the original expert concluded that the questioned signature was not authors. He mainly overvalued the two unmatched styles: one was the last stroke of “关”, and the other was the curve stroke of the middle part of “宾”. The first difference is not essential, the second is apparently different from his ordinary signature style. But in the sample picture (Figure 7), the matched characteristic (as the arrows indicate) that was used in the questioned writing of the middle part of “宾”, is a structure similar to “口”. From the above case, it was proved that when a person wants to write a fluent handwriting with a changed style, he/she generally is using his/her own writing habits, and only changes the placement of characteristics. It should be noted that only limited characteristics can be manipulated like this. For the case of the “郑宾” signature, most habits were matched.

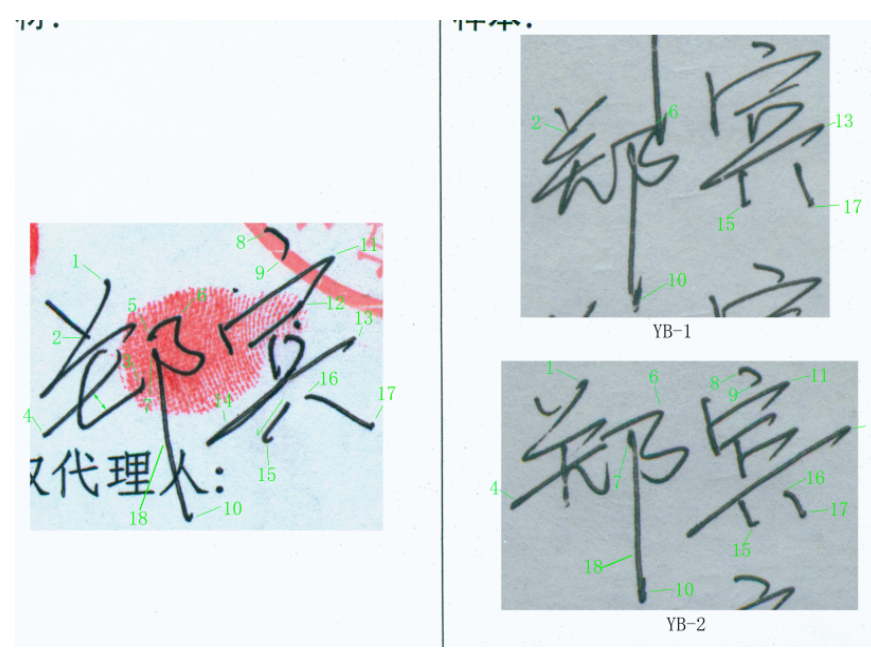

Figure 6. Left side is the signature on questioned document, and the right side is the writing samples collected for comparing after commitment

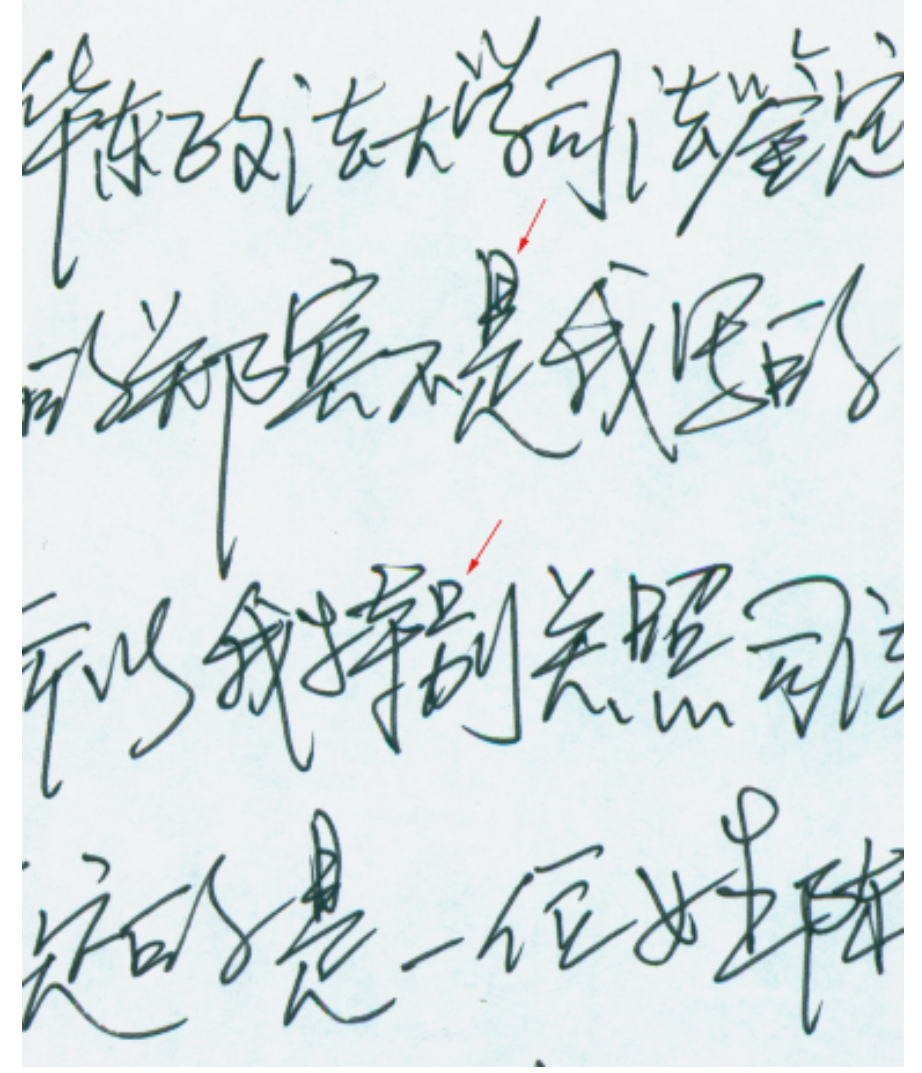

Figure 7. This handwriting sample was collected for comparing after commitment 


\section{Analyze the characteristics and induct right conclusion}

Analyze the source of handwriting: The expert should estimate whether a questioned writing is normal, forged, or disguised, as this is the basic skill of experts. However, it cannot be guarantee that an expert does possess such a skill in practice. In practice, when the expert compares the characteristics, he/she will be indirect in a wrong direction. For example, once he/she regards that the writing of questioned document and sample documents come from the same person, he/she will only compare the same characteristics and ignore the unmatched characteristics and explain the unmatched characteristics to be one's variation. On the contrary, when he/she considers that the questioned writing and sample writing come from different persons, he/she will only compare the different characteristics and treat the matched characteristics as the results of coincidence.

Analyze the habits system by characteristics: Characteristic is the expression of habit, and the total characteristics express the habits system. The expert should find all the matched and unmatched characteristics and analyze them respectively for quantity and quality. Here involve two key aspects: how to define all the characteristics; and how to analyze the quantity and quality.

Analyze the characteristics of the quantity: Finding all the characteristics is very important, as that is the cognition of the entire handwriting. How to find all the characteristics was generally not the experts' consideration, for he/she did not know how to do that. At the beginning of practicing writing, the learner is instructed to write according to various writing procedure and norms, how to write a stroke, arrange the relationship of strokes and the structure of characters. So, the examiner needs to deconstruct the writing motion to the smallest writing motion units, while tracing the stroke path, and to figure out whether there is a characteristic on each writing motion. However, not all the writing motions express habits. Only the individualized writing style is the writing habit. So, a characteristics' individualization value should be considered. For figuring out a writing motion habit, the expert needs to consider the features of handwriting characteristics (as described above): writing norms and convenience. Some norms are easy to comply with, and some are not. If the norm is hard to abide by and one can comply with it in handwriting, this habit is individual among people, and the habit will have a high identification value. If a norm is easy to master but the author does not, the habit will also have a high-level of individualization. Actually, most characteristics deviate from the strict writing norms [30]. Therefore, it is viable to compare the writing with writing norms to define the characteristics and their value [14]

The expert needs to trace the writing path and check the writing motions as follow: the start, process and end of a stroke; the relationships among the strokes; the connect of strokes; the relationship among the parts of a character; and the arrangement styles of characters. He/ she does so to contrast the writing norm and writing convenience for defining characteristics and find out all the characteristics of handwriting under the examination.

Analyze the quality of characteristics: Characteristics quality include two aspects: whether they are normal and how high their individualization values are.

When analyzing whether the writing motion styles are normal, the expert needs to assess whether it is the true expression of the writing habits or comes from an act of disguise or imitation. That can be judged by two factors: writing fluency and writing difficulty. Fluent writing means the writing pressure variate is successive and natural - writing motion doesn't stop or break during the writing process. Writing difficulty refers that a writing habit is hard to fulfill for regular people but not the author. If a writing style is fluent and hard to write for the examiner or others, then the habit comes from the author. If a characteristic appears fluent and easy to write for general people, it may come from the writer, or it may not. If a style is hard to write and not fluent, it may be the result of imitation.

Characteristics' individualization value needs to be estimated by the feature of characteristics described above: writing norms and convenience. For hard norms, characteristics value is positively related to abiding level; for easy norms, the value is positively related to nonabiding level, and vice versa. For convenience, characteristics value is inversely proportional to the degree of writing convenience. This rule is supported by writing characteristics statistics [1].

\section{Draw the conclusion}

After figuring out all the characteristics and their quality, the expert should compare both the matched and unmatched characteristics respectively. When the matched characteristics are the highest in number and have a high degree of individuality compared with the unmatched characteristics, then the conclusion is that the questioned handwriting is attributed to the author, and vice versa. All these methods can be applied in the case of Kunshan Tianyu Trading Co. Ltd v. Duo Xiaoyan (2014) [31] (Figure 8).

In figure 8 , the writing styles marked with green color $1 \sim 5$ lack fluency, so they are imitative writing. Characteristics marked by purple labels are unmatched and difficult to imitate. The most difficult to imitate is the vertical stroke in “艳” marked by “A”. The writing styles of the start, process and end of the stoke are hard to imitate. The difference of pressure variation proves that it is the kind of characteristic difficult to imitate. There are also a lot of writing styles that look like matches but are actually easy writing models, as shown below (Figure 9).

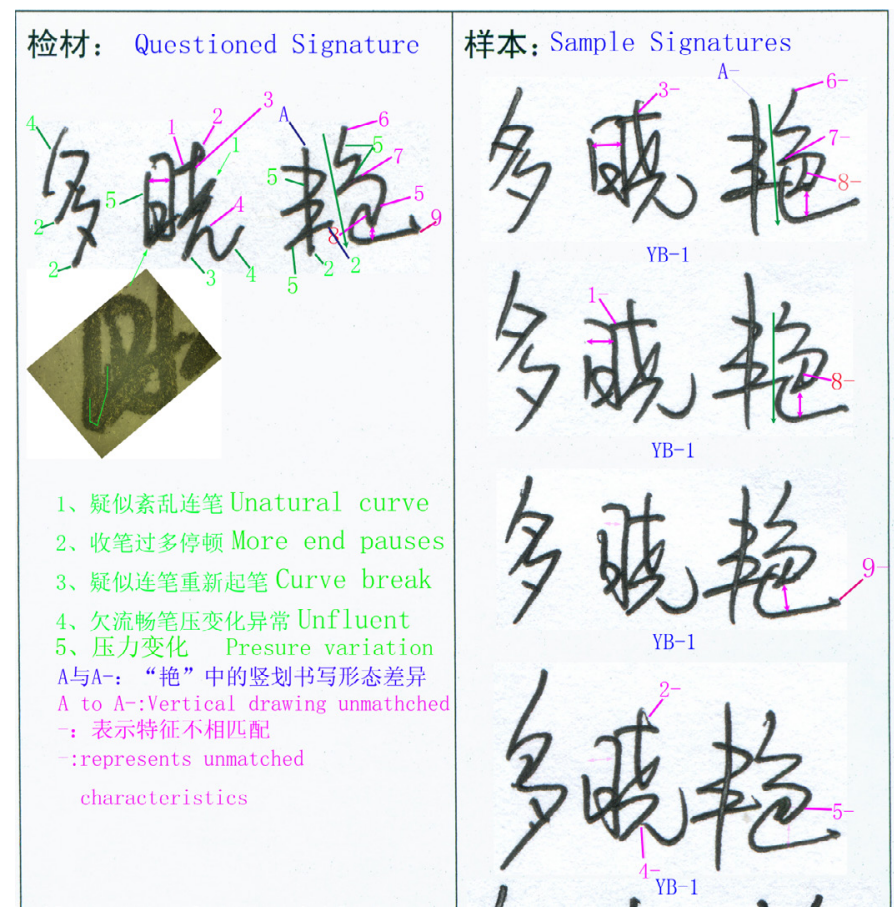

Figure 8. Left side is the signature on questioned document, and the right side is the writing samples collected for comparing after commitment 


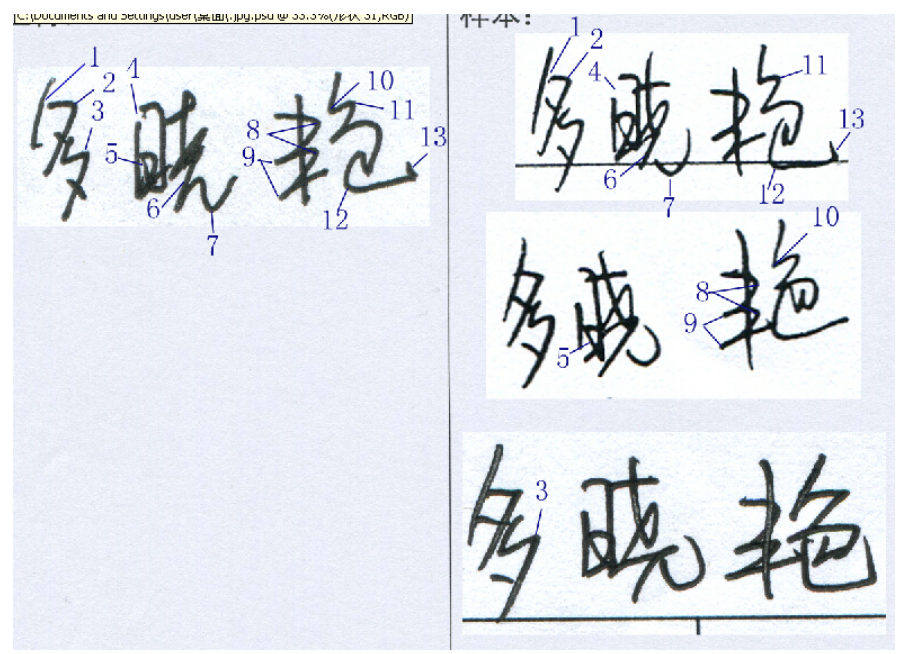

Figure 9. Left side is the signature on questioned document, and the right side is the writing samples collected for comparing after commitment

In figures 8 and 9 , it is shown that the matched or unmatched writing style are both substantial. Analyzing the total individualization of both matched and unmatched characteristics by the writing fluency and difficulty of each characteristic is the key to conduct the right conclusion for this case.

\section{Conclusion}

Valid methods are the key to handwriting identification. Many people regard that writing lacks enough stability that there cannot be reliable methods to analyze it. And there are still many experts treating the writing as a figure and comparing their similarity for identification, for they cannot recognize the stable aspects of the habits system. Any phenomenon has its own rules which can lead to the development of effective methods. Since neurophysiology inherently determines handwriting and the features of writing motions including writing habits, experts could rely on the writing rules and the characteristics of physiology to identify the object. A correct conclusion is built on analyses of quantity and quality of characteristics and the expression of the writing habits system.

\section{References}

1. Hilton O (1992) Scientific examination of questioned documents. CRC press.

2. Werner P, Rosenblum S, Bar-On G, Heinik J, Korczyn A (2006) Handwriting process variables discriminating mild Alzheimer's disease and mild cognitive impairment. $J$ Gerontol B Psychol Sci Soc Sci 61: P228-P236. [Crossref]

3. Talpău F, Tigănescu L, Petruț R (2014) Forensic identification based on handwriting. In: Forensic Science Forum/Forum Criminalistic 7: 2.

4. Huber RA, Headrick AM (1999) Handwriting identification: facts and fundamentals. CRC press.

5. Yu K, Wang Y, Tan T (2004) Writer identification using dynamic features. In Biometric Authentication. Springer Berlin Heidelberg. pp. 512-518.
6. Leedham SSG (2003) A survey of computer methods in forensic handwritten document examination. In: Proceeding the Eleventh International Graphonomics Society Conference, Sccottsdale Arazona pp. 278-281.

7. Osborn S Albert (1910) Questioned Document. The Lawyers' Co-operative Publishing Co

8. Levinson J (2001) Questioned documents: A lawyer's handbook. Academic Press.

9. Kam M, Fielding G, Conn R (1997) Writer identification by professional document examiners. J Forensic Sci 42: 778-786. [Crossref]

10. Kam M, Gummadidala K, Fielding G, Conn R (2001) Signature authentication by forensic document examiners. Journal of Forensic Science 46: 884-888. [Crossref]

11. Daubert v. Merrell Dow Pharmaceuticals, Inc., 509 U.S. 579 (1993).

12. Federal Rules of Evidence > Rule 702. Testimony by Expert Witnesses.

13. Wang G (2003) The New Research about the Theories and Practice of Handwriting Identification. Peking University Press.

14. Wang G (2016) The New Theories of Handwriting Identification - Expert Manual Peking University Press.

15. Cognitive Science (1996) Available from https://plato.stanford.edu/entries/cognitive-science/

16. Diaz-Cabrera M, Ferrer MA, Morales A (2015) Modeling the lexical morphology of western handwritten signatures. PloS one 10: e123254. [Crossref]

17. Terzuolo CA, Viviani P (1980) Determinants and characteristics of motor patterns used for typing. Neuroscience 5: 1085-1103. [Crossref]

18. Smits EJ, Tolonen AJ, Cluitmans L, van Gils M, Conway BA, et al. (2014) Standardized handwriting to assess bradykinesia, micrographia and tremor in Parkinson's disease. PloS one 9: e97614. [Crossref]

19. Harpaz NK, Flash T, Dinstein I (2014) Scale-invariant movement encoding in the human motor system. Neuron 81: 452-462. [Crossref]

20. Horovitz SG, Gallea C, Najee-ullah MA, Hallett M (2013) Functional anatomy of writing with the dominant hand. PloS one 8: e67931. [Crossref]

21. Wang G (2003) The New Research about the Theories and Practice of Handwriting Identification. Peking University Press.

22. Saunders CP, Davis LJ, Buscaglia J (2011) Using automated comparisons to quantify handwriting individuality. J Forensic Sci 56: 683-689. [Crossref]

23. Zhao Wanjie v. Xu Jianping, civil 3349 Xuhui Sh. (Miss. Page. App. 2014)

24. Wang G (2016) The New Theories of Handwriting Identification - Expert Manual Peking University Press.

25. Radu Nicolae Tesan (2014) Scientific Conference in Oradea, on "Forensic GraphologyReferences". Forensic Science NO. 4: 94

26. Goldan E (2013) Rules to Obtain Documents to Compare for Disposal of Handwriting and Signature Expertise/Reguli De Obtinere a Documentelor Pentru Comparatie în Cazul Dispunerii Expertizei Scrisului De Mâna Si Semnaturii. Romanian Journal of Forensic Science 14: 1346-1350.

27. Wang G (2003) Statistical Analysis of Handwriting Characteristics. Forensic Science and Technology 2: 8-11.

28. Committed by Robert C. Brown Member of Dickinson Wright PLLC in court case: PHOENIX-\#221110

29. Shanghai Jinshan HUimin Village Bank v. Shanghai Zhongbao Financial Lease Co. Zheng Bin \& Muhepei, Jinshan SH (Miss Vol and Page App. 2013)

30. Wang G (2003) Statistical Analysis of Handwriting Characteristics. Forensic Science and Technology, 2: 8-11.

31. Kunshan Tianyu Trading Co. Ltd v. Duo Xiaoyan, Kunshan Labor Arbitration Commission (Miss Vol. \& Page. 2014.

Copyright: (C2019 Wang G. This is an open-access article distributed under the terms of the Creative Commons Attribution License, which permits unrestricted use, distribution, and reproduction in any medium, provided the original author and source are credited. 